\title{
Modeling Pulmonary Arterial Hypertension Using Induced Pluripotent Stem Cells
}

\author{
Amer A. Rana, Fedir N. Kiskin, and C.-Hong Chang
}

\begin{abstract}
Heritable pulmonary arterial hypertension belongs to a group of pulmonary vascular diseases for which there is currently no cure other than a heart and lung transplantation. Increased endothelial and smooth muscle cell proliferation leads to vascular remodeling, resulting in increased blood pressure in the lungs which ultimately causes right heart failure. Understanding the molecular mechanisms implicated in the establishment and progression of pulmonary arterial hypertension is hampered due to a lack of disease models, including a limited availability of patient-derived cells and the low penetrance of $B M P R 2$, the major diseaseassociated gene. Induced pluripotent stem cells provide a series of advantages for disease modeling that could potentially allow theses issues to be overcome. These include (1) a limitlessly self-renewing capacity that overcomes the restricted supply of cells from diseased patients, (2) an epigenome that has been wiped of pathogenic modifications which have accumulated during the disease process, (3) an amenability to gene editing which allows the introduction of specific pathogenic mutations in an otherwise isogenic background to facilitate the dissection of the precise contribution of disease-associated genetic mutations, and (4) the generation of isogenic cell, tissue, and organ-specific disease models.
\end{abstract}

\footnotetext{
A. A. Rana (ه)

Division of Respiratory Medicine, Department of Medicine, University of Cambridge, Cambridge, UK

British Heart Foundation Oxford and Cambridge Centre of Regenerative Medicine, Cambridge, UK

British Heart Foundation Cambridge Centre of Research Excellence, Cambridge, UK e-mail: ar332@cam.ac.uk

F. N. Kiskin · C.-H. Chang

Division of Respiratory Medicine, Department of Medicine, University of Cambridge, Cambridge, UK 


\section{Keywords}

Pulmonary arterial hypertension - BMPR2 - Induced pluripotent stem cells · Smooth muscle and endothelial cells $\cdot$ Disease modeling

\subsection{Heritable Pulmonary Arterial Hypertension}

Heritable pulmonary arterial hypertension (HPAH) is a debilitating, often fatal disease caused by increased proliferation and apoptotic resistance of smooth muscle and endothelial cells which drive the vascular remodeling of small pulmonary arterioles and results in their narrowing and obliteration. This causes increased pulmonary vascular resistance and hence increased blood pressure in the pulmonary vessels, eventually leading to right-heart failure [1]. Although currently available therapies have improved life expectancy, they do not prevent disease progression.

\subsubsection{Insights into the Pathobiology of PAH}

Major insights into PAH pathobiology followed the identification of causal heterozygous germline mutations in the gene encoding the bone morphogenetic protein type II receptor (BMPR2) in over $80 \%$ of cases of families with PAH [2]. BMPR2 is a receptor for bone morphogenetic proteins (BMPs), which are members of the transforming growth factor- $\beta$ superfamily. Furthermore, around $20 \%$ of cases of sporadic or idiopathic PAH harbor mutations in BMPR2 [2]. The BMPR2 locus is marked by considerable allelic heterogeneity with over 660 germline variants identified to date. Approximately $70 \%$ of mutations lead to haploinsufficiency due to nonsense-mediated mRNA decay of the mutant transcript. Additional mutations include missense mutations that lead to substitutions in highly conserved amino acids in important functional domains of the receptor [2].

The central role of the BMPR2 pathway in PAH is supported by the identification of causal mutations in other members of this pathway including ACVRL1, ENG, GDF2 (BMP9), and SMAD9 [3]. In addition, loss of BMPR2 signaling appears to play a critical role in non-genetic forms of PAH in humans and in preclinical rodent models. Furthermore, treatment with the selective BMPR2/ALK1 ligand BMP9 has been shown to increase lung endothelial BMPR2 expression and reverse PAH in rodent models [4]

\subsubsection{Reduced Penetrance of BMPR2 in PAH}

An important observation in heritable PAH is that BMPR2 mutations exhibit low penetrance (approximately $20-30 \%$ in families), suggesting a requirement for additional environmental or genetic triggers necessary for disease initiation and progression [5]. Interestingly, identical twins carrying mutations in $B M P R 2$, where only 
one sibling presents with PAH, have been identified [6]. Together, these argue that environmental factors must have a critical role in disease establishment, and in some contexts, these may be more important than the presence of particular genetic modifiers of BMPR2.

Insights into potential environmental factors that interact with BMPR2 to cause PAH have come from animal models free of genetic mutations in BMPR2, including lipopoly-saccharide-, monocrotaline-, Sugen-5416/hypoxia-, and chronic hypoxiainduced mouse and rat models, as well as from mice with lung-specific overexpression of tumor necrosis factor ( $\mathrm{TNF} \alpha$ ) [7]. However, a common and important feature of these non-genetic models of PAH is the general reduction in BMPR2 at the protein level, which further supports a central role of BMPR2 in the establishment of PAH. In recent work from our labs, we have dissected the mechanism of how inflammatory signaling in the form of TNF $\alpha$ can cause a reduction in BMPR2 signaling [8]. This is achieved by via the shedding of BMPR2 extracellular domain at the cell surface, which reduces intact receptor expression, as well as by the transcriptional repression of $B M P R 2$, which reduces the total overall expression of BMPR2 in cells. Taken together, these changes ultimately lead to hyper-proliferation of pulmonary artery smooth muscle cells.

\subsection{Modeling Pulmonary Arterial Hypertension with Induced Pluripotent Stem Cells}

The limited availability of human tissue from PAH patients with end-stage disease and only at the time of transplantation has hampered progress in understanding the initiation and progression of PAH. In addition, mouse knockout or transgenic models of BMPR2 deficiency fail to fully recapitulate the pathology observed in humans [7]. Hence, there is a pressing need for alternative human models of PAH to accelerate the development of new treatments for this devastating disease.

Induced pluripotent stem cells (iPSCs) provide major opportunities for disease modeling because they can be derived from specific patients, possess a limitless ability to self-renew, differentiate into all somatic cell lineages, and are amenable to targeted genetic engineering [9]. Their epigenetic memory is also reset during the reprogramming process, wiping epigenetic remodeling marks accumulated during the disease process and therefore potentially providing a naïve non-diseased system which can be used to induce a disease state and thus elucidate hitherto unknown mechanisms of disease progression.

However, an important consideration is whether iPSC-derived cells resemble the relevant adult cell types closely enough. In the case of PAH, the goal is to mimic pulmonary arterial endothelial and distal smooth muscle cells. For example, fundamental differences in responses to hypoxia exist between the pulmonary vasculature and the systemic vasculature and also between the BMP responsiveness of proximal and peripheral (distal) portions of the pulmonary vascular branch.

While systemic arteries relax in response to tissue hypoxia in order to improve blood flow and oxygen supply in hypoxic tissues, pulmonary arteries respond by 
contracting, thereby diverting blood flow from poorly ventilated regions toward the more oxygenated areas of the lungs [10]. Furthermore, previous work has demonstrated that in contrast to proximal cells, peripheral PASMCs are not growthsuppressed and exhibit reduced apoptosis when treated with BMP4 [11].

\subsubsection{Embryological Origins of the Pulmonary Vasculature}

In order to differentiate iPSCs toward pulmonary vascular lineages we must consider the embryological origins of the pulmonary vascular cells and the developmental pathways that have directed them toward their specific fate. For example, the differences in responsiveness to hypoxia and BMP signaling might be due to cells of the pulmonary vasculature having different embryological origins. Lineage tracing has shown that four major embryonic lineages contribute to the pulmonary vasculature: (1) Wnt1+ neural crest, which contributes to the SMCs at the root of the pulmonary artery, (2) cardiopulmonary progenitors (CPPs), which contribute ECs and SMCs to the proximal pulmonary artery and vein, (3) WT1+ mesothelium, which surrounds the lung and contributes widely to both ECs and SMCs throughout the vasculature, and (4) lateral plate mesoderm, which contributes to the PASMCs in peripheral pulmonary arteries [12]. The ability to recapitulate specific lineages and their vascular derivatives is likely to be critical for generating a high-fidelity model of PAH.

\subsubsection{Current iPSC Models of PAH}

Our group was the first to publish work on reprogramming patient-derived cells carrying BMPR2 mutations [13]. Since then, other reports have used iPSC-derived cells to gain insights into PAH.

West et al. [14] used patient-derived iPSCs carrying mutations in BMPR2 to generate mesenchymal and endothelial-like cells. The authors noted an increase in Wnt signaling in these cells and found this was also the case in adult BMPR2 mutant fibroblasts and in human tissue. However, although the mesenchymal cells expressed some SMC markers, these cells were unable to model the characteristic hyperproliferative phenotype observed in PASMCs. This highlights the important consideration that iPSC-derived cells must approximate closely enough the relevant adult cell types.

Sa et al. [15] performed RNA-seq on iPSC-derived endothelial cells (iPSC-ECs) and pulmonary artery endothelial cells (PAECs) with and without BMPR2 mutations and observed that cell adhesion and migration were decreased in cells carrying BMPR2 mutations compared to controls. This work also showed that KISS1 was upregulated in $B M P R 2$ mutant cells compared to wild-type cells.

$\mathrm{Gu}$ et al. [16] reiterated the cell adhesion and cell migration defect in iPSCECs but went on to look at why not all BMPR2 mutation carriers develop 
PAH. Their approach was to take three different families, in which all individuals carried mutations in $B M P R 2$, and then compare the phenotype and transcriptomes of iPSC-ECs of individuals within each family to see if there was a difference between those family members that present with PAH (FPAH) and those that did not (unaffected mutation carriers or UMC). Through this they found that apoptosis was higher in FPAH compared with UMC, while cell adhesion was reduced in both FPAH and UMC. This suggested that genetic modifiers might give UMCs a cell survival advantage. Through transcriptomic analysis, BIRC3, a gene implicated in cell survival, was found to be reduced in FPAH compared to UMC. Taken together, the authors concluded that genetic background can influence whether or not an individual will develop PAH. However, as discussed earlier, regardless of the initial instigator (genetic or non-genetic) of PAH, all patients ultimately show a reduction in BMPR2 expression and signaling. Thus, the question of what BMPR2 is doing in disease is fundamental and remained unanswered.

We recently addressed this issue using iPSCs to model arterial ECs and distal PASMCs where we were able to transition cells from a naïve state lacking diseaseassociated cellular phenotypes to a diseased state, showing that a BMPR2 mutation per se is necessary and sufficient for the establishment of some but not all PAHassociated cellular phenotypes in iPSC-ECs and iPSC-SMCs [21]. Omics analysis of the changes associated with this transition will reveal potentially novel and/or druggable pathways to prevent or reverse PAH disease progression.

\subsection{Future Direction and Clinical Implications}

Primary future uses of iPSCs include drug screening and the generation of human 3D multi-tissue constructs of the pulmonary system for disease modeling. Protocols to generate endothelial cells (ECs), smooth muscle cells, and pericytes from iPSCs have been established [17, 18], and advanced protocols to generate cells more akin to pulmonary vascular cells are underway. Based on these protocols, functional evaluations in each cell type between normal and disease-carrying subjects can be performed to provide a powerful platform for cell type-specific drug discovery. Considering the importance of cell-cell communication during disease progression, modeling diseases will require using more than one cell type. Although it is possible to co-culture iPSCderived ECs and perivascular cells [19], the highly specialized and complex nature of the pulmonary vascular bed poses challenges for constructing a 3D pulmonary-specific vasculature. In addition, investigating merely vascular cells might lead us to misinterpret pulmonary vascular disease mechanisms. Although animal models have been widely used to model diseases, they are expensive, time-consuming, and often do not fully recapitulate human pathology. iPSC-derived organ-like tissue, termed 'organoids', might eventually replace animal models [20]. Whilst liver and kidney organoids develop with vasculatures, current lung organoids lack vascular populations [20]. Developing better lung organoids or pulmonary artery-on-a-chip technologies would provide an advanced platform to model pulmonary vascular diseases. 
Acknowledgments This research was funded by the British Heart Foundation (BHF) (project grant PG/14/31/30786 and FS/13/51/30636), the Cambridge NIHR Biomedical Research Centre, the Dinosaur Trust, PHA UK, and the Robert McAlpine Foundation. AAR would also like to acknowledge support from the BHF Centre of Regenerative Medicine, Oxford and Cambridge (RM/13/3/30159), the BHF Centre for Research Excellence (RE/13/6/30180), and the BHF IPAH cohort grant (SP/12/12/29836). We apologize to those authors whose work we were unable to cite due to space limitations.

\section{References}

1. Humbert M, Morrell NW, Archer SL, Stenmark KR, MacLean MR, Lang IM, et al. Cellular and molecular pathobiology of pulmonary arterial hypertension. J Am Coll Cardiol. 2004;43:13S-24S.

2. Machado RD, Southgate L, Eichstaedt CA, Aldred MA, Austin ED, Best DH, et al. Pulmonary Arterial Hypertension: A Current Perspective on Established and Emerging Molecular Genetic Defects. Hum Mutat. 2015;36(12):1113-27.

3. Ma L, Chung WK. The role of genetics in pulmonary arterial hypertension. J Pathol. 2017;241(2):273-80.

4. Long L, Ormiston ML, Yang X, Southwood M, Gräf S, Machado RD, et al. Selective enhancement of endothelial BMPR-II with BMP9 reverses pulmonary arterial hypertension. Nat Med. 2015;21(7):777-85.

5. Larkin EK, Newman JH, Austin ED, Hemnes AR, Wheeler L, Robbins IM, et al. Longitudinal analysis casts doubt on the presence of genetic anticipation in heritable pulmonary arterial hypertension. Am J Respir Crit Care Med. 2012;186(9):892-6.

6. Ormiston ML, Southgate L, Treacy C, Pepke-Zaba J, Trembath RC, Machado RD, et al. Assessment of a pulmonary origin for blood outgrowth endothelial cells by examination of identical twins harboring a BMPR2 mutation. Am J Respir Crit Care Med. 2013;188(2):258-60.

7. Stenmark KR, Meyrick B, Galie N, Mooi WJ, McMurtry IF. Animal models of pulmonary arterial hypertension: the hope for etiological discovery and pharmacological cure. Am J Physiol Lung Cell Mol Physiol. 2009;297(6):L1013-32.

8. Hurst LA, Dunmore BJ, Long L, Crosby A, Al-Lamki R, Deighton J, et al. TNF $\alpha$ drives pulmonary arterial hypertension by suppressing the BMP type-II receptor and altering NOTCH signalling. Nat Commun. 2017;8:14079.

9. Shi Y, Inoue H, Wu JC, Yamanaka S. Induced pluripotent stem cell technology: a decade of progress. Nat Rev Drug Discov. 2017;16(2):115-30.

10. Ward JP, McMurtry IF. Mechanisms of hypoxic pulmonary vasoconstriction and their roles in pulmonary hypertension: new findings for an old problem. Curr Opin Pharmacol. 2009;9(3):287-96.

11. Yang X, Long L, Southwood M, Rudarakanchana N, Upton PD, Jeffery TK, et al. Dysfunctional Smad signaling contributes to abnormal smooth muscle cell proliferation in familial pulmonary arterial hypertension. Circ Res. 2005;96(10):1053-63.

12. Majesky MW. Developmental basis of vascular smooth muscle diversity. Arterioscler Thromb Vasc Biol. 2007;27(6):1248-58.

13. Geti I, Ormiston ML, Rouhani F, Toshner M, Movassagh M, Nichols J, et al. A practical and efficient cellular substrate for the generation of induced pluripotent stem cells from adults: blood-derived endothelial progenitor cells. Stem Cells Transl Med. 2012;1(12):855-65.

14. West JD, Austin ED, Gaskill C, Marriott S, Baskir R, Bilousova G, et al. Identification of a common Wnt-associated genetic signature across multiple cell types in pulmonary arterial hypertension. Am J Physiol Cell Physiol. 2014;307(5):C415-30.

15. Sa S, Gu M, Chappell J, Shao NY, Ameen M, Elliott KA, et al. Induced Pluripotent Stem Cell Model of Pulmonary Arterial Hypertension Reveals Novel Gene Expression and Patient Specificity. Am J Respir Crit Care Med. 2017;195(7):930-41. 
16. Gu M, Shao NY, Sa S, Li D, Termglinchan V, Ameen M, et al. Patient-specific iPSC-derived endothelial cells uncover pathways that protect against pulmonary hypertension in BMPR2 mutation carriers. Cell Stem Cell. 2017;20(4):490-504.e5.

17. Kiskin FN, Chang C-H, Huang CJZ, Kwieder B, Cheung C, Dunmore BJ, Serrano F, Sinha S, Morrell NW, Rana AA. Contributions of mutations and extrinsic factors to cellular phenotypes of pulmonary arterial hypertension revealed by induced pluripotent stem cell modeling. Am J Respir Crit Care Med. 2018;198(2):271-5.

18. Orlova VV, van den Hil FE, Petrus-Reurer S, Drabsch Y, Ten Dijke P, Mummery CL. Generation, expansion and functional analysis of endothelial cells and pericytes derived from human pluripotent stem cells. Nat Protoc. 2014;9(6):1514-31.

19. Cheung C, Bernardo AS, Trotter MW, Pedersen RA, Sinha S. Generation of human vascular smooth muscle subtypes provides insight into embryological origin-dependent disease susceptibility. Nat Biotechnol. 2012;30(2):165-73.

20. Heydarkhan-Hagvall S, Helenius G, Johansson BR, Li JY, Mattsson E, Risberg B. Co-culture of endothelial cells and smooth muscle cells affects gene expression of angiogenic factors. J Cell Biochem. 2003;89(6):1250-9.

21. Dye BR, Hill DR, Ferguson MA, Tsai YH, Nagy MS, Dyal R, et al. In vitro generation of human pluripotent stem cell derived lung organoids. Elife. 2015;4. https://doi.org/10.7554/ eLife.05098.

Open Access This chapter is licensed under the terms of the Creative Commons Attribution 4.0 International License (http://creativecommons.org/licenses/by/4.0/), which permits use, sharing, adaptation, distribution and reproduction in any medium or format, as long as you give appropriate credit to the original author(s) and the source, provide a link to the Creative Commons license and indicate if changes were made.

The images or other third party material in this chapter are included in the chapter's Creative Commons license, unless indicated otherwise in a credit line to the material. If material is not included in the chapter's Creative Commons license and your intended use is not permitted by statutory regulation or exceeds the permitted use, you will need to obtain permission directly from the copyright holder.

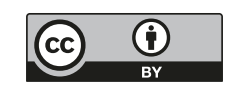

